“C 2017 IEEE. Personal use of this material is permitted. Permission from IEEE must be obtained for all other uses, in any current or future media, including reprinting/republishing this material for advertising or promotional purposes, creating new collective works, for resale or redistribution to servers or lists, or reuse of any copyrighted component of this work in other works." 


\title{
Model Predictive Switching Pattern Control for Current Source Converters with Space-Vector-Based Selective Harmonic Elimination
}

\author{
Hang Gao, Bin Wu, Fellow, IEEE, Dewei(David) Xu, Member, IEEE, Ricardo P. Aguilera, Member, \\ IEEE, Pablo Acuña, Member, IEEE
}

\begin{abstract}
This paper presents a model predictive switching pattern control (MPSPC) for a current source converter (CSC), which achieves superb low-order harmonics elimination performance in steady state, and improved transient responses. Based on a proposed space vector based selective harmonic elimination (SHE) method and prediction of load current at the next sampling instant, MPSPC prefers to following a pre-calculated SHE-PWM pattern in steady state, and governing the CSC through a model predictive control (MPC) approach during transients. In comparison with existing schemes, the advantages of MPSPC are threefold: First, quantization error, introduced by a constant sampling frequency in MPC and degrading steady-state low-order harmonic elimination, is mitigated in the proposed scheme. Second, there is no weighting factor in the cost function, as used in existing schemes. Last, MPSPC is totally realized based on one-step prediction, which simplifies the structure of the scheme. Both simulation and experimental results verify the steady-state and dynamic performance of MPSPC with different SHE-PWM patterns.
\end{abstract}

Index Terms - Current source converter; selective harmonic elimination modulation; model predictive switching pattern control; cost function.

\section{INTRODUCTION}

$\mathrm{R}$ ecently, in medium-voltage (MV) motor drives, pulse-width-modulated (PWM) current source converters (CSCs) appear to be a good alternative to voltage source converters (VSCs) due to their advantages including four-quadrant operation, reliable short-circuit protection, machine-friendly waveforms, and reduced cost [1]. As Fig. 1

Manuscript received August 26, 2016; accepted October 3.

H. Gao, B. Wu, and D. (D.) Xu are with the Department of Electrical and Computer Engineering, Ryerson University, Toronto, ON M5B 2K3, Canada (e-mail: h2gao@ee.ryerson.ca; bwu@ee.ryerson.ca; dxu@ryerson.ca)

R. P. Aguilera is with the School of Electrical, Mechanical and Mechatronic Systems, University of Technology Sydney, Sydney, NSW 2007, Australia (e-mial: raguilera@ieee.org)

P. Acuña is with the Australian Energy Research Institute and the School of Electrical Engineering and Telecommunications, The University of New South Wales, Syndney, NSW 2052, Australia (e-mail: pablo.acuna@unsw.edu.au)

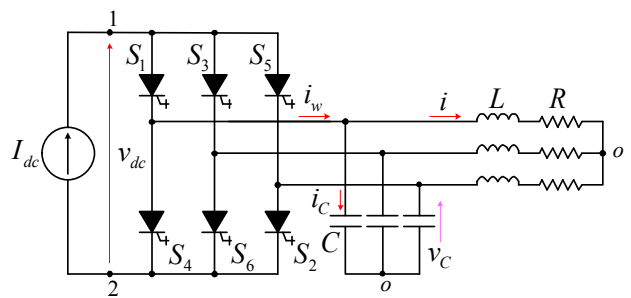

Fig. 1. Topology of a single-bridge current source converter.

shows, the CSC is composed of six symmetrical gate-commutated thyristors (SGCTs) with reverse voltage blocking capability. On the dc side is an ideal current source, which is usually obtained by a current source rectifier (CSR) and a series inductor in practice. A three-phase capacitor must be connected at the output of the CSC to assist the commutations of switching devices, and also used as a filter.

For MV applications, low switching frequencies are preferred in order to minimize the switching losses of switching devices. There are mainly two modulation techniques for the CSC focusing on switching frequency reduction, which includes space vector modulation (SVM) and selective harmonic elimination PWM (SHE-PWM) [1, 2]. Nevertheless, lowering the switching frequency increases low-order harmonics in PWM current generated by SVM, resulting in higher harmonic losses. So far, SHE-PWM is always considered as one approach to fulfill the operating requirements of MV motor drives. As an offline modulation method, the optimal switching angles used in SHE-PWM are pre-calculated to eliminate the selected low-order harmonics, and stored in a look-up table (LUT). Industrial applications of MV motor drives impose increasingly stringent performance requirements. Traditionally, SHE-PWM is normally realized in a modulator driven by a very slow control loop, which ensures stable steady-state performance. However, this leads to a poor dynamic performance, and to transient distortion of load currents when the operating point is changed or when transitions between different SHE-PWM patterns occur. Moreover, capacitor currents need to be compensated when calculating output current references for the CSC. To reduce the sensitivity to the variation of capacitor current, the capacitor transient response is normally neglected in the calculated 
capacitor current, which also leads to the degraded dynamic performance of the CSC [3]. Furthermore, no matter in grid-connected or motor-drive applications, the load normally presents inductive behavior. The inductor in the load constructs a LC resonance tank with the three-phase capacitor. The LC resonance can be easily excited during transients, which possibly causes overcurrent and overvoltage $[4,5]$. Generally, implementing a closed-loop control scheme for power converters using SHE-PWM is not a trivial task, especially for the CSC.

Recently, model predictive control (MPC) has emerged as a promising alternative to govern power converters. MPC based approach generally provides more superb dynamic responses compared to traditional controls [6]. In previous literature, MPC has been introduced for two-level and multilevel VSCs $[7,8]$ in grid-connected and motor-drive applications $[9,10]$. MPCs for current source inverter (CSI) with three-phase RL load are proposed in $[11,12]$. In [13], MPC for CSR is presented, in which dc current regulation and reactive power control are realized by an associated cost function. However, the existing schemes are just suitable for low power CSC with high switching frequency. Besides, the resulting switching actuations during every sampling interval remain unknown, which leads to variable switching frequencies in steady state.

Up to now, for MV application, MPCs with very low switching frequency have been proposed, either. In [14], model predictive direct torque control (MPDTC) is proposed for MV motor drives, through which both torque and stator flux magnitude are kept in their respective bonds, and the switching frequency can be reduced compared to standard DTC. Whereas, since the torque and flux are directly regulated, the distortion of stator current is not taken into consideration in this scheme. To minimize the THD of the stator current for a given switching frequency, model predictive pulse pattern control (MP3C) is proposed in [15]. A prediction horizon of multi-steps in time is used, and the switching instants of the pulse pattern are shifted, such that a stator flux error is corrected within this horizon. From the end of the horizon onwards, offline calculated optimal pulse pattern (OPP) is assumed to be used in steady state. Though MP3C can improve dynamic performance, and maintain OPP output in steady state, the method requires multistep prediction, which complicates the realization, and increases the calculation burden. Similarly, multistep MPC has been shown to have the potential to achieve a performance similar to that of optimized voltage patterns in [16]. However, the main concern of multistep methods still focuses on the higher calculation requirement, compared to one-step approach. MPC, generating an SHE-like pattern has been proposed in [17]. This scheme is based on a sliding discrete Fourier transform (SDFT) that calculates voltage harmonics in real time, then it uses this information to mitigate unwanted harmonics. This method still involves huge calculation, since a large sampling window (one fundamental period at least) is used to properly calculate these harmonics. Besides, dynamic performance is not considered in this method. A SHE MPC has been proposed in [18], through which the output voltage of multilevel VSC follows SHE PWM pattern in steady state, and the VSC generates optimally selected output voltage states by MPC during transients to ensure improved dynamic response. However, there are mainly two drawbacks existing in this scheme. One is that due to a constant sampling frequency used in it, quantization effect is introduced, and leads to the error between the practically applied switching angles and the optimally calculated ones, which degrades its low-order harmonic elimination performance. Since a weighting factor is used in the cost function to associate two variables with different units (predicted load currents and SHE base output voltage states) together, the tuning of weighting factor has direct influence on the final output performance.

In this work, a model predictive switching pattern control (MPSPC) for the CSC is presented, which eliminates quantization errors in steady state, and avoids the use of weighting factor in comparison with the state-of-the-art schemes. MPSPC is realized based on a proposed space vector representation of SHE-PWM pattern for the CSC. During a constant sampling interval, a saw-tooth carrier is applied to generate SHE-PWM waveform through a quasi-modulation process. Besides, at every sampling instant, according to the selected space vectors and calculated dwell time for SHE-PWM pattern, the load current at the end of this sampling interval can be predicted. Then, the error between the load current reference and its predicted value can be obtained. If the error is smaller than a predefined maximum limitation, the output PWM current just follows the SHE-PWM pattern, which means that SHE-PWM can satisfy the requirement of output performance. On the other hand, with the error larger than the maximum limitation, which usually appears during transient process, the applied output current vector during this sampling interval is optimally selected through a MPC method, which achieves improved dynamic performance with shorter settling time and suppressed LC resonance. At the next sampling instant, this procedure is repeated with new measurements to keep the load current tracking its reference. The proposed scheme has been verified based on different SHE-PWM patterns for the CSC.

Compared to the SHE-PWM modulator in tradition control, MPSPC improves the dynamic performance, and preserves the SHE-PWM waveform in the steady state. Compared to the state-of-the-art MPC approaches, the benefits of MPSPC are threefold. First, with a saw-tooth carrier based quasi-modulation process in MPSPC, there is no quantization error, as mentioned in [18], any more, which totally maintains the selective low-order harmonic elimination performance. Second, there is no weighting factor selection issue, as in [14, 18], since only predicted load current and its reference appear in the cost function. Though a maximum limitation of load current error needs to be defined, it has more significant physical meaning, and is easier to be selected, in comparison with a weighting factor. Last but not least, MPSPC only uses a one-step or horizon-one prediction, not like the multistep predictions in [14-16]. The one-step prediction leads to a simple structure, and reduces the complexity of the proposed scheme. With MPSPC, the switching frequency can be kept 


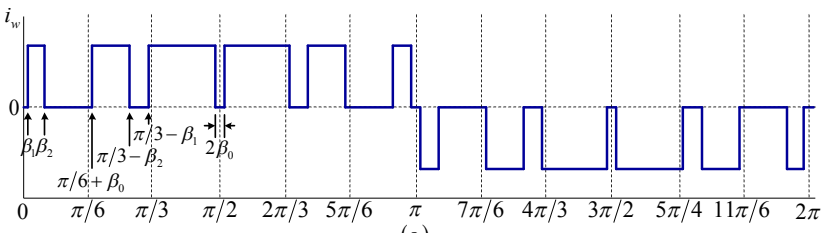

(a)

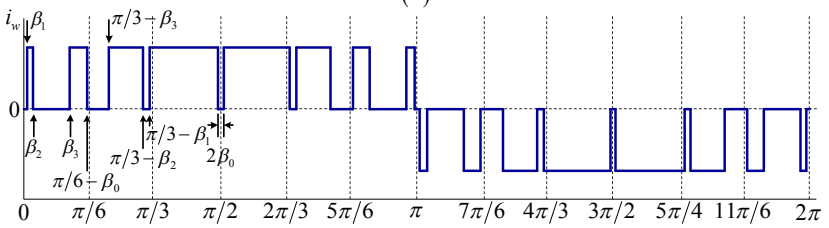

(b)

Fig. 2. SHE PWM waveforms: (a) six pulses per half-cycle and (b) eight pulses per half-cycle.

constant and at a very low value in steady state. During transients, the CSC is governed by MPC to achieve improved dynamic performance, which achieves superior output performance for MV applications.

This paper is organized as follows. Section II summarizes the background of SHE-PWM for the CSC, while Section III proposes a space vector based $\mathrm{SHE}$ as the foundation of MPSPC. Section IV describes the details of the proposed scheme. Simulation results using in a high power MV CSC (1 $\mathrm{MW} / 4160 \mathrm{~V} / 17.3 \mathrm{~A})$ and experimental results in a low power prototype $(3 \mathrm{~kW} / 208 \mathrm{~V} / 8.33 \mathrm{~A})$ are presented in Section $\mathrm{V}$ and VI, respectively. Finally, some conclusions are drawn in Section VII.

\section{SHE-PWM FOR CSC}

SHE-PWM pattern should meet switching constraints of the $\mathrm{CSC}$, that is at any instant of time there are only two switching devices conducting, one in the top half and the other in the bottom half of the bridge. Normally there are two commonly used SHE-PWM pattern for the CSC, namely six pulses per half cycle for output frequency around $60 \mathrm{~Hz}$, and eight pulses per half cycle for output frequency around $50 \mathrm{~Hz}$. With these two kinds of PWM patterns, the switching frequencies of switching devices in the CSC are just about $360 \mathrm{~Hz}$ or $400 \mathrm{~Hz}$ [1]. Fig. 2 shows typical SHE-PWM waveforms for the CSC. In Fig. 2 (a), there are six switching angles in the first $\pi / 2$ period of six-pulse waveform. However, only three out of the six angles, $\beta_{1}, \beta_{2}$, and $\beta_{0}$, are independent, which can be used to eliminate two harmonics and in the meanwhile provide and adjustable modulation index. Similarly, the waveform with eight pulses per half cycle is depicted in Fig. 2 (b), in which there are only four independent angles, $\beta_{1}, \beta_{2}, \beta_{3}$, and $\beta_{0}$. With these four angles, three selected harmonics can be eliminated, and modulation index can be adjusted, either.

The PWM current waveform in Fig. 2 can be expressed in Fourier series as

$i_{w}(\omega t)=\sum_{n=1}^{\infty} a_{n} \sin (n \omega t)$

where
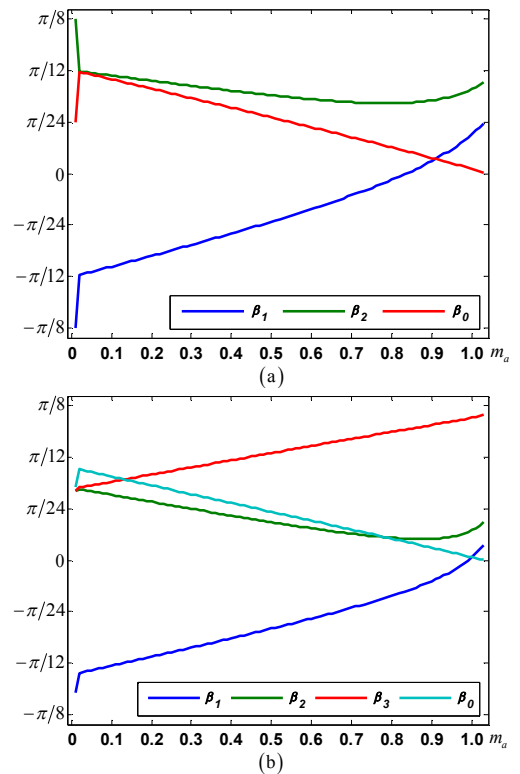

Fig. 3. Independent angle solutions for SHE (a) six pulse per half cycle and (b) eight pulse per half cycle.

$a_{n}=\frac{4}{\pi} \int_{0}^{\frac{\pi}{2}} i_{w}(\omega t) \sin (n \omega t) d(n \omega t)$

Here six-pulse waveform is used as an example. In order to eliminate two dominant low-order harmonics such as the $5^{\text {th }}$ and $7^{\text {th }}$, and adjust modulation index, the following system of equations is defined as

$$
\left\{\begin{array}{l}
F_{1}=\cos \left(5 \beta_{1}\right)-\cos \left(5 \beta_{2}\right)+\cos \left(5\left(\pi / 6+\beta_{0}\right)\right)-\cos \left(5\left(\pi / 3-\beta_{2}\right)\right) \\
+\cos \left(5\left(\pi / 3-\beta_{1}\right)\right)-\cos \left(5\left(\pi / 2-\beta_{0}\right)\right)=0 \\
F_{2}=\cos \left(11 \beta_{1}\right)-\cos \left(11 \beta_{2}\right)+\cos \left(11\left(\pi / 6+\beta_{0}\right)\right)-\cos \left(11\left(\pi / 3-\beta_{2}\right)\right) \\
+\cos \left(11\left(\pi / 3-\beta_{1}\right)\right)-\cos \left(11\left(\pi / 2-\beta_{0}\right)\right)=0 \\
F_{3}=(4 / \pi)\left[\cos \beta_{1}-\cos \beta_{2}+\cos \left(\pi / 6+\beta_{0}\right)\right. \\
\left.-\cos \left(\pi / 3-\beta_{2}\right)+\cos \left(\pi / 3-\beta_{1}\right)-\cos \left(\pi / 2-\beta_{0}\right)\right]-m_{a}=0
\end{array}\right.
$$

where $m_{a}$ is the modulation index, given by

$m_{a}=\frac{\hat{I}_{w 1}}{I_{d c}}$

in which $\hat{I}_{w 1}$ is the peak fundamental-frequency component of the PWM current, and $I_{d c}$ is the average dc current.

Similarly, for eight-pulse waveform with four independent angles, four equations can also be established to eliminate three dominant harmonics, such as the $5^{\text {th }}, 7^{\text {th }}$, and $11^{\text {th }}$, and adjust modulation index.

The nonlinear and transcendental equation of (3) can be solved by a number of numerical methods [19], one of which is the Newton-Raphson iteration algorithm. At various modulation indexes, independent angles for six- and eight-pulse waveforms have been calculated and the solutions are shown in Fig. 3.

In Fig. 3, no matter for six-pulse or eight-pulse waveform, the maximum modulation index is around 1.02, at which $\beta_{0}$ becomes zero and the notch in the center of the half-cycle PWM waveform disappears. For a given value of $\beta_{1}, \beta_{2}$, and $\beta_{0}$, 

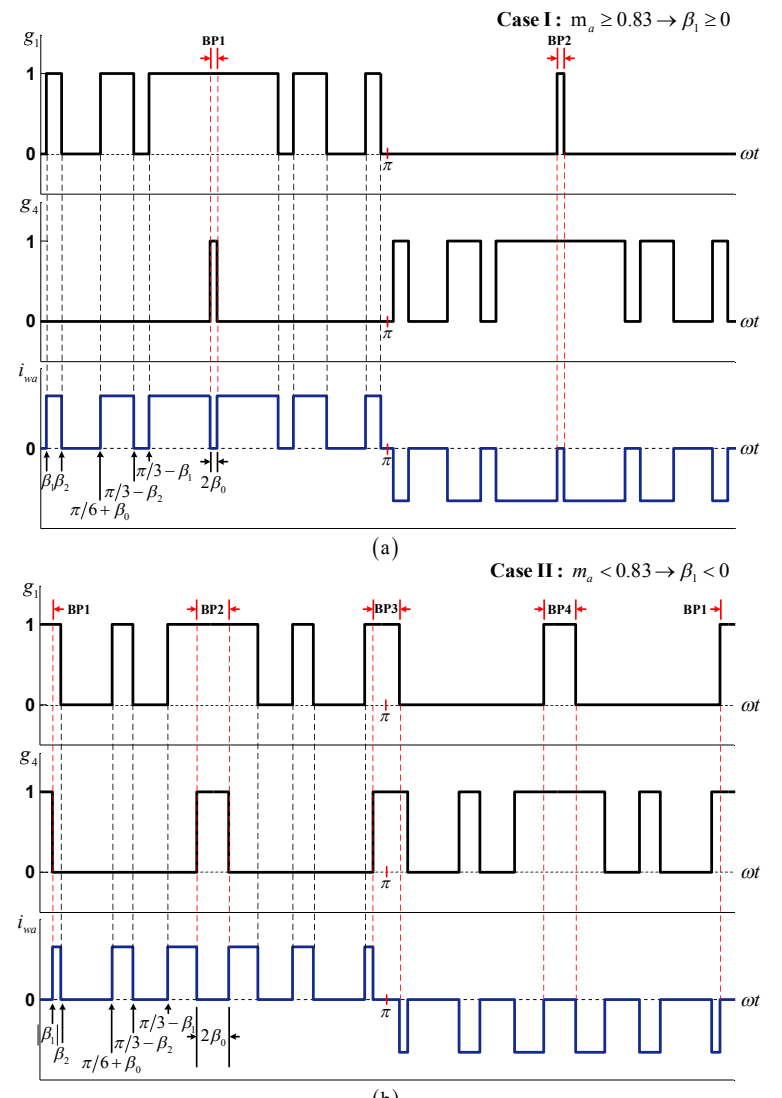

(b)

Fig. 4. Phase A gate signals for six-pulse waveform: (a) Case I and (b) Case II.

the gate signals for the switching devices in the CSC can be arranged. As Fig. 3 (a) shows, for six-pulse waveform, when $m_{a}$ is lower than $0.83, \beta_{1}$ is smaller than 0 , which means that there are two cases of gate signal arrangements for six-pulse waveform. In Fig. 4, $g_{1}$ and $g_{4}$ are the gate signals for $S_{1}$ and $S_{4}$ in the CSC. $g_{1}$ is composed of six pulses, of which one is the bypass pulse, defined between $3 \pi / 2-\beta_{0}$ and $3 \pi / 2+\beta_{0}$. If $\beta_{1}$ is larger than 0 , there are only two bypass intervals, which are caused by bypass pulses, as Fig. 4 (a) shows. If $\beta_{1}$ is smaller than 0 , there are four bypass intervals per cycle. The bypass intervals, BP2 and BP4, are created by bypass pulses while the other two, BP1 and BP3, are due to the overlapping of the gate signals, as Fig. 4 (b) shows.

Similarly, as Fig. 3 (b) shows, for eight-pulse waveform, when $m_{a}$ is lower than $0.98, \beta_{1}$ is smaller than 0 . When $m_{a}$ is between 0.89 and $0.98,\left|\beta_{1}\right|$ is smaller than $\beta_{1}$. If $m_{a}$ is between 0.26 and $0.89,\left|\beta_{1}\right|$ is larger than $\beta_{2}$, but smaller than $\beta_{3}$. With $m_{a}$ smaller than $0.26,\left|\beta_{1}\right|$ is finally larger than $\beta_{3}$. These four situations lead to four cases of gate signal arrangements for eight-pulse waveform. As Fig. 5 shows, $g_{1}$ is composed of eight pulses. Similarly, there is one bypass pulse, defined between $3 \pi / 2-\beta_{0}$ and $3 \pi / 2+\beta_{0}$. If $\beta_{1}$ is larger than 0 , there are only two bypass intervals, which are generated by bypass pulses, as Fig. 5 (a) shows. If $\beta_{1}$ is smaller than 0 ,

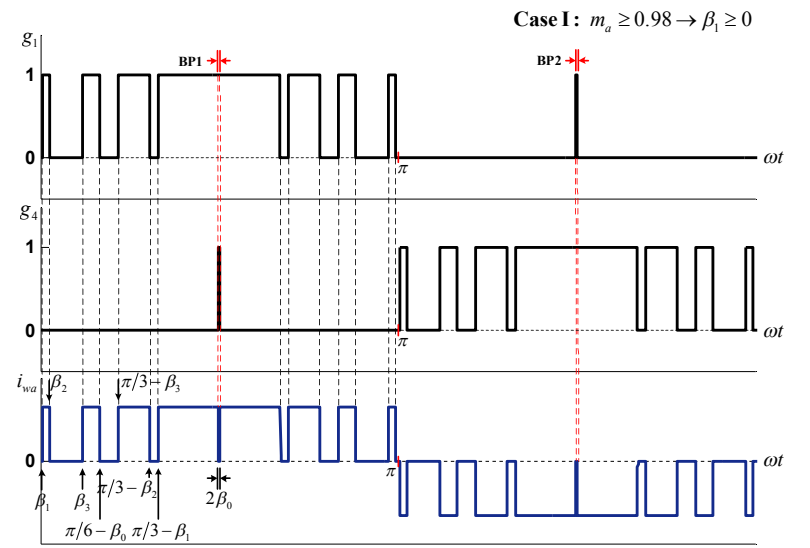

(a)
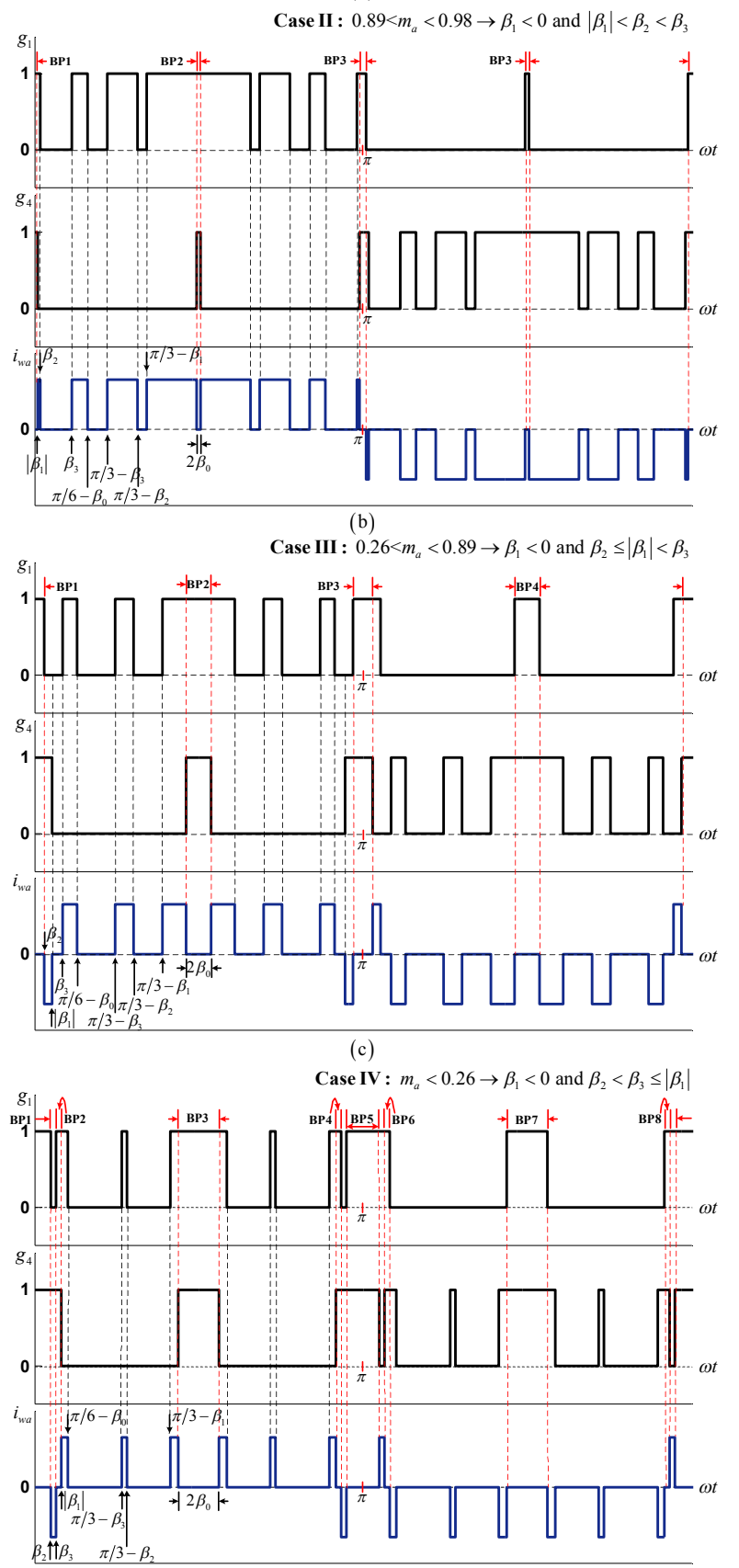

(d)

Fig. 5. Phase A gating signals for eight pulse waveform: (a) Case I, (b) Case II, (c) Case III, and (d) Case IV. 


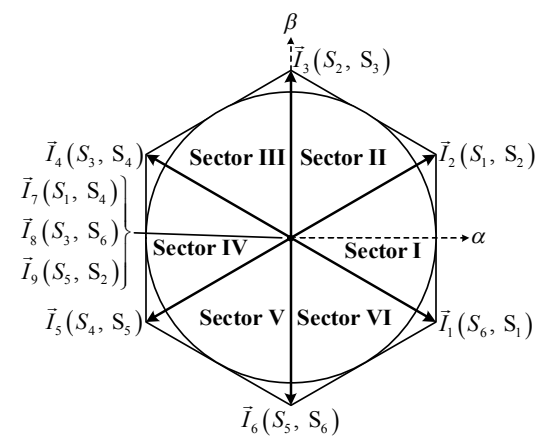

Fig. 6. Space vector diagram of CSC.

and $\left|\beta_{1}\right|$ is no larger than $\beta_{3}$, meaning case II and III, there are four bypass intervals per cycle. The bypass intervals, BP2 and BP4, are created by bypass pulses while the other two, BP1 and $\mathrm{BP} 3$, are due to the overlapping of the gate signals, as Fig. 5 (b) and (c) illustrate. If $\beta_{1}$ is smaller than 0 , and $\left|\beta_{1}\right|$ is larger than $\beta_{3}$, there are eight bypass intervals per cycle. The bypass intervals, BP3 and BP7, are created by bypass pulses while the other six are due to the overlapping of the gate signals, as Fig. 5 (d) shows.

\section{SPACE VECTOR BASED SHE}

In traditional modulator, the switching angles for SHE are directly used to generated PWM waveform. However, a fixed sampling frequency, $f_{s}$, is normally used in MPC. The sampling process introduces a quantization error on the pre-calculated switching angles, as discussed in [18]. As a consequence, the elimination of undesired low-order harmonics may be compromised since the quantized angles may differ from the pre-calculated ones. In order to ensure an acceptable low-order harmonic elimination performance, a high sampling frequency needs to be used, which increases the requirement on calculating capability of digital signal processor (DSP).

As the foundation of MPSPC, a space vector based SHE with a fixed sampling frequency, but without quantization error is proposed here. The space vector representation of SHE waveform will be introduced first, then the realization of space vector based SHE will be described in details.

\section{A. Space Vector Representation of SHE}

At any instant of time, SHE waveforms for the CSC satisfy the switching constraints, which means that one corresponding space vector is applied. Fig. 6 shows the space vector diagram of the CSC. Hence, based on the switching signals of six switching devices in the CSC, SHE waveform can be represented in space vector form. Here the SHE waveforms during time interval between 0 and $\pi / 3$ are selected as an example. For six-pulse waveforms, shown in Fig. 4, since there are two cases of gate signal arrangements based on modulation index value, the space vector sequence during the selected interval also has two kinds of patterns, as Fig. 7 shows. Similarly, as Fig. 5 shows, since there are four gate signal

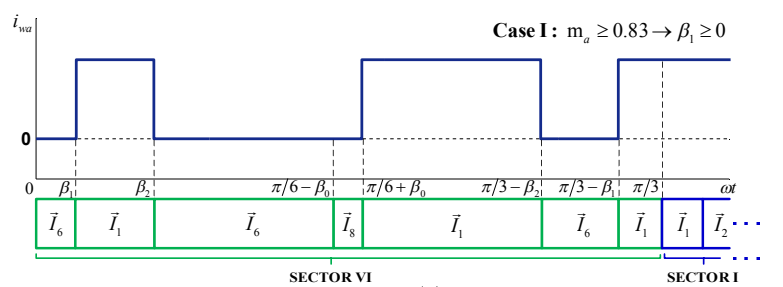

(a)

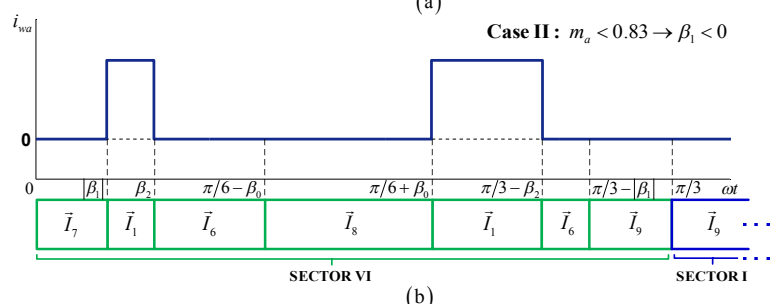

Fig. 7. Space vector sequence for six-pulse waveform: (a) case I and (b) case II.

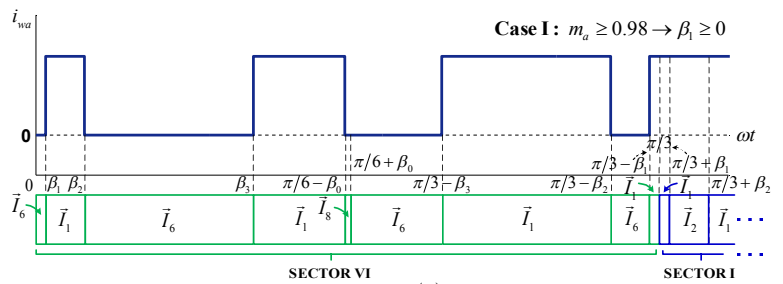

(a)

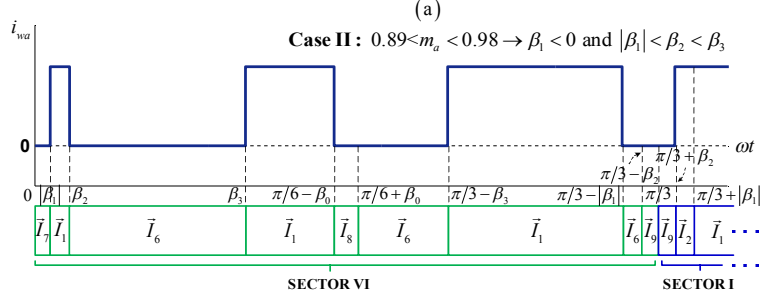

(b)

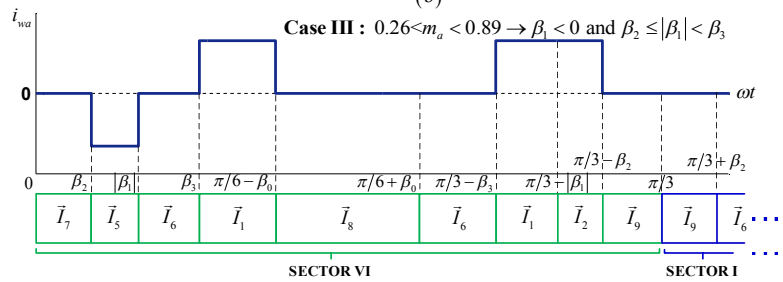

(c)

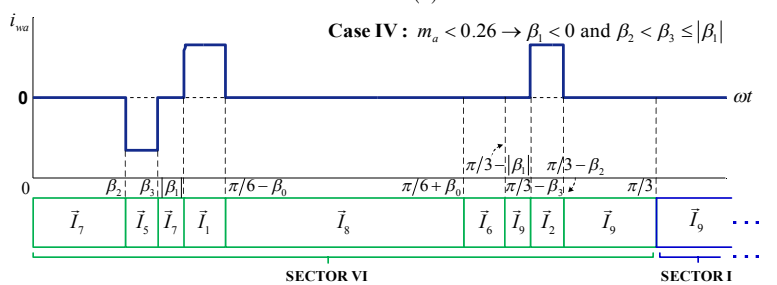

(d)

Fig. 8. Space vector sequence for eight-pulse waveform: (a) case I, (b) case II, and (c) case III, and (d) case IV.

arrangements for eight-pulse waveforms based on different modulation indexes, the four kinds of space vector sequences are shown in Fig. 8.

It can be found from Fig. 7 and Fig. 8 that with $\beta_{1}$ decreasing from positive value to negative value, more zero-state vectors appear in the space vector sequence due to the overlapping operation. Besides, the dwell time for each space vector can be calculated based on difference between the 
TABLE I

SPACE VECTOR SEQUENCES FOR SIX-PULSE WAVEFORM

\begin{tabular}{cll}
\hline \hline Sector No. & \multicolumn{1}{c}{ Case I } & \multicolumn{1}{c}{ Case II } \\
\hline Sector I & $\vec{I}_{1} \rightarrow \vec{I}_{2} \rightarrow \vec{I}_{1} \rightarrow \vec{I}_{7}$ & $\vec{I}_{9} \rightarrow \vec{I}_{2} \rightarrow \vec{I}_{1} \rightarrow \vec{I}_{7}$ \\
& $\rightarrow \vec{I}_{2} \rightarrow \vec{I}_{1} \rightarrow \vec{I}_{2}$ & $\rightarrow \vec{I}_{2} \rightarrow \vec{I}_{1} \rightarrow \vec{I}_{8}$ \\
& $\vec{I}_{2} \rightarrow \vec{I}_{3} \rightarrow \vec{I}_{2} \rightarrow \vec{I}_{9}$ & $\vec{I}_{8} \rightarrow \vec{I}_{3} \rightarrow \vec{I}_{2} \rightarrow \vec{I}_{9}$ \\
Sector II & $\rightarrow \vec{I}_{3} \rightarrow \vec{I}_{2} \rightarrow \vec{I}_{3}$ & $\rightarrow \vec{I}_{3} \rightarrow \vec{I}_{2} \rightarrow \vec{I}_{7}$ \\
& $\vec{I}_{3} \rightarrow \vec{I}_{4} \rightarrow \vec{I}_{3} \rightarrow \vec{I}_{8}$ & $\vec{I}_{7} \rightarrow \vec{I}_{4} \rightarrow \vec{I}_{3} \rightarrow \vec{I}_{8}$ \\
Sector III & $\rightarrow \vec{I}_{4} \rightarrow \vec{I}_{3} \rightarrow \vec{I}_{4}$ & $\rightarrow \vec{I}_{4} \rightarrow \vec{I}_{3} \rightarrow \vec{I}_{9}$ \\
& $\vec{I}_{4} \rightarrow \vec{I}_{5} \rightarrow \vec{I}_{4} \rightarrow \vec{I}_{7}$ & $\vec{I}_{9} \rightarrow \vec{I}_{5} \rightarrow \vec{I}_{4} \rightarrow \vec{I}_{7}$ \\
Sector IV & $\rightarrow \vec{I}_{5} \rightarrow \vec{I}_{4} \rightarrow \vec{I}_{5}$ & $\rightarrow \vec{I}_{5} \rightarrow \vec{I}_{4} \rightarrow \vec{I}_{8}$ \\
& $\vec{I}_{5} \rightarrow \vec{I}_{6} \rightarrow \vec{I}_{5} \rightarrow \vec{I}_{9}$ & $\vec{I}_{8} \rightarrow \vec{I}_{6} \rightarrow \vec{I}_{5} \rightarrow \vec{I}_{9}$ \\
Sector V & $\rightarrow \vec{I}_{6} \rightarrow \vec{I}_{5} \rightarrow \vec{I}_{6}$ & $\rightarrow \vec{I}_{6} \rightarrow \vec{I}_{5} \rightarrow \vec{I}_{7}$ \\
& $\vec{I}_{6} \rightarrow \vec{I}_{1} \rightarrow \vec{I}_{6} \rightarrow \vec{I}_{8}$ & $\vec{I}_{7} \rightarrow \vec{I}_{1} \rightarrow \vec{I}_{6} \rightarrow \vec{I}_{8}$ \\
Sector VI & $\rightarrow \vec{I}_{1} \rightarrow \vec{I}_{6} \rightarrow \vec{I}_{1}$ & $\rightarrow \vec{I}_{1} \rightarrow \vec{I}_{6} \rightarrow \vec{I}_{9}$ \\
\hline & &
\end{tabular}

TABLE II

SPACE VECTOR SEQUENCES FOR EIGHT-PULSE WAVEFORM

\begin{tabular}{|c|c|c|c|c|}
\hline $\begin{array}{c}\text { Sector } \\
\text { No. }\end{array}$ & Case I & Case II & Case III & Case IV \\
\hline Sector I & $\begin{array}{l}\vec{I}_{1} \rightarrow \vec{I}_{2} \rightarrow \vec{I}_{1} \\
\rightarrow \vec{I}_{2} \rightarrow \vec{I}_{7} \rightarrow \vec{I}_{1} \\
\rightarrow \vec{I}_{2} \rightarrow \vec{I}_{1} \rightarrow \vec{I}_{2}\end{array}$ & $\begin{array}{l}\vec{I}_{9} \rightarrow \vec{I}_{2} \rightarrow \vec{I}_{1} \\
\rightarrow \vec{I}_{2} \rightarrow \vec{I}_{7} \rightarrow \vec{I}_{1} \\
\rightarrow \vec{I}_{2} \rightarrow \vec{I}_{1} \rightarrow \vec{I}_{8}\end{array}$ & $\begin{array}{l}\vec{I}_{9} \rightarrow \vec{I}_{6} \rightarrow \vec{I}_{1} \\
\rightarrow \vec{I}_{2} \rightarrow \vec{I}_{7} \rightarrow \vec{I}_{1} \\
\rightarrow \vec{I}_{2} \rightarrow \vec{I}_{3} \rightarrow \vec{I}_{8}\end{array}$ & $\begin{array}{l}\vec{I}_{9} \rightarrow \vec{I}_{6} \rightarrow \vec{I}_{9} \\
\rightarrow \vec{I}_{2} \rightarrow \vec{I}_{7} \rightarrow \vec{I}_{1} \\
\rightarrow \vec{I}_{8} \rightarrow \vec{I}_{3} \rightarrow \vec{I}_{8}\end{array}$ \\
\hline Sector II & $\begin{array}{l}\vec{I}_{2} \rightarrow \vec{I}_{3} \rightarrow \vec{I}_{2} \\
\rightarrow \vec{I}_{3} \rightarrow \vec{I}_{9} \rightarrow \vec{I}_{2} \\
\rightarrow \vec{I}_{3} \rightarrow \vec{I}_{2} \rightarrow \vec{I}_{3}\end{array}$ & $\begin{array}{l}\vec{I}_{8} \rightarrow \vec{I}_{3} \rightarrow \vec{I}_{2} \\
\rightarrow \vec{I}_{3} \rightarrow \vec{I}_{9} \rightarrow \vec{I}_{2} \\
\rightarrow \vec{I}_{3} \rightarrow \vec{I}_{2} \rightarrow \vec{I}_{7}\end{array}$ & $\begin{array}{l}\vec{I}_{8} \rightarrow \vec{I}_{1} \rightarrow \vec{I}_{2} \\
\rightarrow \vec{I}_{3} \rightarrow \vec{I}_{9} \rightarrow \vec{I}_{2} \\
\rightarrow \vec{I}_{3} \rightarrow \vec{I}_{4} \rightarrow \vec{I}_{7}\end{array}$ & $\begin{array}{l}\vec{I}_{8} \rightarrow \vec{I}_{1} \rightarrow \vec{I}_{8} \\
\rightarrow \vec{I}_{3} \rightarrow \vec{I}_{9} \rightarrow \vec{I}_{2} \\
\rightarrow \vec{I}_{2} \rightarrow \vec{I}_{4} \rightarrow \vec{I}_{7}\end{array}$ \\
\hline $\begin{array}{l}\text { Sector } \\
\text { III }\end{array}$ & $\begin{array}{l}\vec{I}_{3} \rightarrow \vec{I}_{4} \rightarrow \vec{I}_{3} \\
\rightarrow \vec{I}_{4} \rightarrow \vec{I}_{8} \rightarrow \vec{I}_{3} \\
\rightarrow \vec{I}_{4} \rightarrow \vec{I}_{3} \rightarrow \vec{I}_{4}\end{array}$ & $\begin{array}{l}\vec{I}_{3} \rightarrow \vec{I}_{4} \rightarrow \vec{I}_{3} \\
\rightarrow \vec{I}_{4} \rightarrow \vec{I}_{8} \rightarrow \vec{I}_{3} \\
\rightarrow \vec{I}_{4} \rightarrow \vec{I}_{3} \rightarrow \vec{I}_{4}\end{array}$ & $\begin{array}{l}\vec{I}_{7} \rightarrow \vec{I}_{2} \rightarrow \vec{I}_{3} \\
\rightarrow \vec{I}_{4} \rightarrow \vec{I}_{8} \rightarrow \vec{I}_{3} \\
\rightarrow \vec{I}_{4} \rightarrow \vec{I}_{5} \rightarrow \vec{I}_{9}\end{array}$ & $\begin{array}{l}\vec{I}_{7} \rightarrow \vec{I}_{2} \rightarrow \vec{I}_{7} \\
\rightarrow \vec{I}_{4} \rightarrow \vec{I}_{8} \rightarrow \vec{I}_{3} \\
\rightarrow \vec{I}_{9} \rightarrow \vec{I}_{5} \rightarrow \vec{I}_{9}\end{array}$ \\
\hline $\begin{array}{l}\text { Sector } \\
\text { IV }\end{array}$ & $\begin{array}{l}\vec{I}_{4} \rightarrow \vec{I}_{5} \rightarrow \vec{I}_{4} \\
\rightarrow \vec{I}_{5} \rightarrow \vec{I}_{7} \rightarrow \vec{I}_{4} \\
\rightarrow \vec{I}_{5} \rightarrow \vec{I}_{4} \rightarrow \vec{I}_{5}\end{array}$ & $\begin{array}{l}\vec{I}_{9} \rightarrow \vec{I}_{5} \rightarrow \vec{I}_{4} \\
\rightarrow \vec{I}_{5} \rightarrow \vec{I}_{7} \rightarrow \vec{I}_{4} \\
\rightarrow \vec{I}_{5} \rightarrow \vec{I}_{4} \rightarrow \vec{I}_{8}\end{array}$ & $\begin{array}{l}\vec{I}_{9} \rightarrow \vec{I}_{3} \rightarrow \vec{I}_{4} \\
\rightarrow \vec{I}_{5} \rightarrow \vec{I}_{7} \rightarrow \vec{I}_{4} \\
\rightarrow \vec{I}_{5} \rightarrow \vec{I}_{6} \rightarrow \vec{I}_{8}\end{array}$ & $\begin{array}{l}\vec{I}_{9} \rightarrow \vec{I}_{3} \rightarrow \vec{I}_{9} \\
\rightarrow \vec{I}_{5} \rightarrow \vec{I}_{7} \rightarrow \vec{I}_{4} \\
\rightarrow \vec{I}_{8} \rightarrow \vec{I}_{6} \rightarrow \vec{I}_{8}\end{array}$ \\
\hline $\begin{array}{l}\text { Sector } \\
\text { IV }\end{array}$ & $\begin{array}{l}\vec{I}_{5} \rightarrow \vec{I}_{6} \rightarrow \vec{I}_{5} \\
\rightarrow \vec{I}_{6} \rightarrow \vec{I}_{9} \rightarrow \vec{I}_{5} \\
\rightarrow \vec{I}_{6} \rightarrow \vec{I}_{5} \rightarrow \vec{I}_{6}\end{array}$ & $\begin{array}{l}\vec{I}_{8} \rightarrow \vec{I}_{6} \rightarrow \vec{I}_{5} \\
\rightarrow \vec{I}_{6} \rightarrow \vec{I}_{9} \rightarrow \vec{I}_{5} \\
\rightarrow \vec{I}_{6} \rightarrow \vec{I}_{5} \rightarrow \vec{I}_{7}\end{array}$ & $\begin{array}{l}\vec{I}_{8} \rightarrow \vec{I}_{4} \rightarrow \vec{I}_{5} \\
\rightarrow \vec{I}_{6} \rightarrow \vec{I}_{9} \rightarrow \vec{I}_{5} \\
\rightarrow \vec{I}_{6} \rightarrow \vec{I}_{1} \rightarrow \vec{I}_{7}\end{array}$ & $\begin{array}{l}\vec{I}_{8} \rightarrow \vec{I}_{4} \rightarrow \vec{I}_{8} \\
\rightarrow \vec{I}_{6} \rightarrow \vec{I}_{9} \rightarrow \vec{I}_{5} \\
\rightarrow \vec{I}_{7} \rightarrow \vec{I}_{1} \rightarrow \vec{I}_{7}\end{array}$ \\
\hline $\begin{array}{c}\text { Sector } \\
\text { VI }\end{array}$ & $\begin{array}{l}\vec{I}_{6} \rightarrow \vec{I}_{1} \rightarrow \vec{I}_{6} \\
\rightarrow \vec{I}_{1} \rightarrow \vec{I}_{8} \rightarrow \vec{I}_{6} \\
\rightarrow \vec{I}_{1} \rightarrow \vec{I}_{6} \rightarrow \vec{I}_{1}\end{array}$ & $\begin{array}{l}\vec{I}_{7} \rightarrow \vec{I}_{1} \rightarrow \vec{I}_{6} \\
\rightarrow \vec{I}_{1} \rightarrow \vec{I}_{8} \rightarrow \vec{I}_{6} \\
\rightarrow \vec{I}_{1} \rightarrow \vec{I}_{6} \rightarrow \vec{I}_{9}\end{array}$ & $\begin{array}{l}\vec{I}_{7} \rightarrow \vec{I}_{5} \rightarrow \vec{I}_{6} \\
\rightarrow \vec{I}_{1} \rightarrow \vec{I}_{8} \rightarrow \vec{I}_{6} \\
\rightarrow \vec{I}_{1} \rightarrow \vec{I}_{2} \rightarrow \vec{I}_{9}\end{array}$ & $\begin{array}{l}\vec{I}_{7} \rightarrow \vec{I}_{5} \rightarrow \vec{I}_{7} \\
\rightarrow \vec{I}_{1} \rightarrow \vec{I}_{8} \rightarrow \vec{I}_{6} \\
\rightarrow \vec{I}_{9} \rightarrow \vec{I}_{2} \rightarrow \vec{I}_{9}\end{array}$ \\
\hline
\end{tabular}

start and end terminals of one space vector, which changes with respect to different calculated optimal angles. Another thing is that during the selected duration, $\vec{I}_{6}$ and $\vec{I}_{1}$ mainly appear in the above sequences as active-state vectors, which indicates that the reference vector for SHE waveform generation locates in SECTOR VI of the space vector domain in Fig. 6. Since the sequences in SECTOR VI has been analyzed, the principle and regulation can be extended to other sectors. The dwell time for each space vector in the same case can also be used for other sectors. All the sequences in different sectors for six-pulse and eight-pulse waveforms have been listed in Table I and Table II, respectively.

\section{B. Realization of Space Vector Based SHE}

In most applications, load currents are directly regulated by power converters. For the CSC, the output current reference can be calculated based on the summation of load current reference and capacitor current compensation. The output

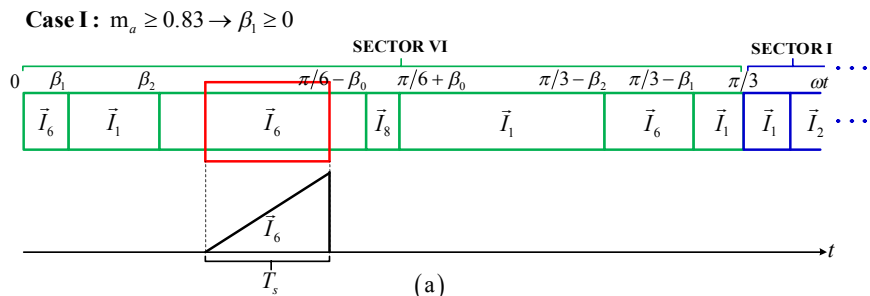

(a)

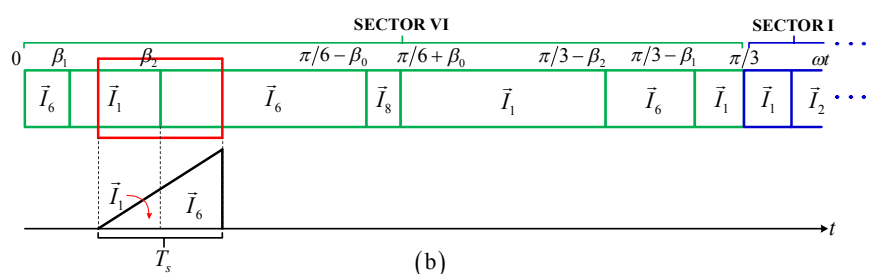

(b)

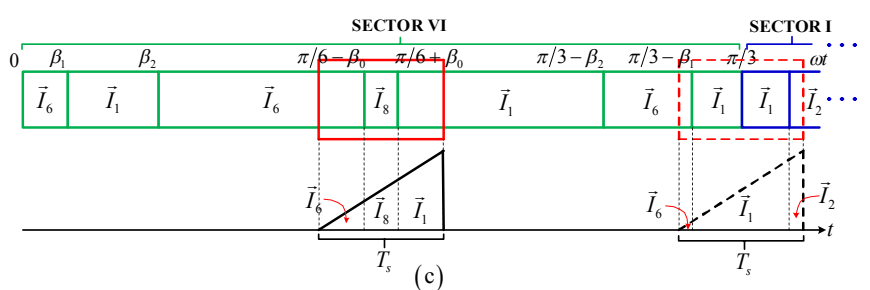

Fig. 9. Space vectors during one sampling interval: (a) one vector, (b) two vectors, and (c) three vectors.

current reference can be expressed in space vector form as

$\vec{i}_{w}^{\text {ref }}=\hat{I}_{w}^{\text {ref }} e^{j \theta_{w}^{\text {ref }}}$

where $\hat{I}_{w}^{\text {ref }}$ is the magnitude of output current reference, and $\theta_{w}^{\text {ref }}$ is the angle of output current reference vector in space vector domain. Based on (4), the modulation index can be calculated. Based on the angle, the sector, in which the output current reference vector locates, can be determined. Then, the angle of the output current reference vector, with respect to the corresponding sector, can be obtained. According to the modulation index, the optimal angles can be selected from the pre-calculated look-up table, then the space vector sequence, corresponding to the calculated modulation index and specified sector, can be determined. Finally, based on the angle of output current reference vector, with respect to the corresponding vector, the location of the start point of the sampling interval can be obtained in one sector sequence. Since a fixed sampling frequency is used here, sampling interval is a constant value. Normally, for MPC, the sampling frequency is selected as high as $20 \mathrm{kHz}$, even $50 \mathrm{kHz}$ in some cases. Here, taken into consideration of calculation capability of most state-to-art DSPs, $10 \mathrm{kHz}$ sampling frequency is selected. With a constant sampling frequency and the information on the start point of the sampling interval, the location of the end point of the sampling interval can also be calculated.

Here the space vector sequence in case I of six-pulse waveforms is selected as an example. During the sampling interval, there are possibly three scenarios of space vector combinations, as Fig. 9 shows. In Fig. 9 (a), only one vector is applied during the whole sampling interval. As Fig. 9 (b) shows, if the sampling interval locates between two vectors, the applied vector needs to be changed once during the interval. There is another situation in which the applied vector, during 
one sampling interval, needs to be changed twice, as Fig. 9 (c) depicts. Since $\beta_{1}$ and $\beta_{0}$ can be very close to 0 at some specified modulation index, the sampling interval is possibly divided into three parts for three space vectors. In fact, there are only two start point locations of sampling interval, which possibly leads to this situation. When the sampling interval locates around the half center of the PWM waveform, the sampling interval may contain three vectors, as the red rectangular shown in Fig. 9 (c). When the start point of the sampling interval locates near the end of the sector, the end point of the sampling interval may locate in the next sector. With a small value of $\beta_{1}$, the sampling interval also possibly contains three space vectors, as the red dotted rectangular shown in Fig. 9 (c). For eight-pulse SHE waveform, the three situations also exist with very small values of $\beta_{1}$ and $\beta_{0}$

- Moreover, with higher sampling frequency and smaller sampling interval, the above discussion is still available.

Based on a specified space vector sequence and locations of the start point and end point of one sampling interval, space vectors and their respective dwell time during one sampling interval can be determined. With a saw-tooth carrier, of which frequency is equal to sampling frequency, the selected space vectors can be modulated in one sampling interval with their corresponding dwell time, as Fig. 9 shows. Since space vectors during one sampling interval can maximum be changed twice, the quasi-modulation method during one sampling interval can be easily realized based on DSP or FPGA. Moreover, with the space vector based SHE, there is no quantization error introduced by a constant sampling frequency, since there is no compromise on the practically applied switching angles in comparison with pre-calculated ones.

Format and save your graphic images using a suitable graphics processing program that will allow you to create the images as PostScript (PS), Encapsulated PostScript (EPS), or Tagged Image File Format (TIFF), sizes them, and adjusts the resolution settings. If you created your source files in one of the following you will be able to submit the graphics without converting to a PS, EPS, or TIFF file: Microsoft Word, Microsoft PowerPoint, Microsoft Excel, or Portable Document Format (PDF).

\section{PROPOSED MPSPC}

With space vector based SHE, SHE waveform can be generated without quantization error, even at a constant sampling frequency. However, the generated SHE waveform can just ensure steady state performance. It is caused by two reasons, one is that when calculating output current references for the CSC, capacitor current compensations only consider steady state capacitor currents with transient responses totally ignored, the other is that the essence of SHE is not an online modulation scheme which only guarantees the steady state performance. In this section, a MPSPC scheme is proposed to

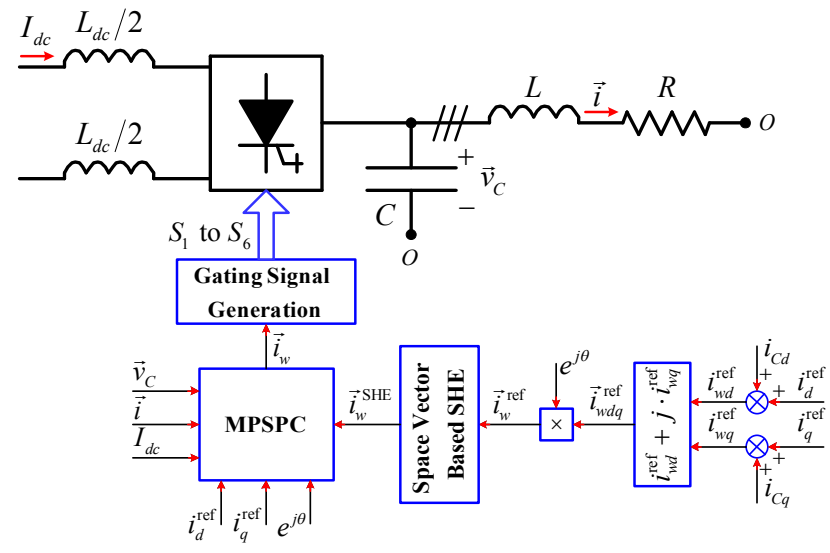

Fig. 10. Block diagram of proposed MPSPC for CSC.

improve the transient performance of space vector based SHE. In steady state, the output PWM waveform follows SHE pattern. During transients, the output waveform of the CSC will be naturally changed, and regulated by MPC for better transient performance. Compared to existing methods, there is no weighting factor selection issue, such as the one used to associate the predicted load currents and SHE based output voltage states together in one cost function, as presented in [18]. Fig. 10 shows the control block diagram of proposed MPSPC for the CSC with a RL load. In Fig. 10, $\vec{i}_{w}^{\text {SHE }}$ represents the selected space vectors based on SHE-PWM pattern during one sampling interval. The selected space vectors and their respective dwell time are sent into MPSPC stage, then the final output current space vector, $\vec{i}_{w}$, can be determined, and used to generate gate signals for switching devices in the CSC.

\section{A. Steady State Reference Calculation}

With load current references, output current references in $d q$-axis synchronous frame can be calculated as

$\left\{\begin{array}{l}i_{w d}=i_{d}^{\text {ref }}+i_{C d} \\ i_{w q}=i_{q}^{\text {ref }}+i_{C q}\end{array}\right.$

in which

$\left\{\begin{array}{l}i_{C d}=-\omega C v_{C q} \\ i_{C q}=\omega C v_{C d}\end{array}\right.$

It can be found from (7) that transient responses of capacitor currents are totally ignored. With the calculated output current references, output current reference vector in $\alpha \beta$-stationary frame can be expressed as

$\vec{i}_{w}^{\text {ref }}=\left(i_{w d}+j \cdot i_{w q}\right) e^{j \theta}$

where $\theta$ is the instantaneous space angle of the synchronous frame, which are determined based on different applications. In motor drive applications, $\theta$ is determined based on the estimated rotor flux vector. In grid-connected rectifier or inverter applications, a phase-lock-loop (PLL) is used to obtain the angle of the grid voltage vector, which is the angle of the synchronous frame in these cases. 


\section{B. Principle of Proposed MPSPC}

Here, for simplicity, the CSC, which is connected to a simple RL load, is selected as an example. The continuous-time output model of CSC can be expressed as

$\dot{\mathbf{x}}=\mathbf{A x}+\mathbf{B u}$

in which

$$
\mathbf{A}=\left[\begin{array}{rr}
0 & -\frac{1}{C} \\
\frac{1}{L} & -\frac{R}{L}
\end{array}\right] \text { and } \mathbf{B}=\left[\begin{array}{c}
\frac{1}{C} \\
0
\end{array}\right]
$$

where $\mathbf{X}$ is state variable, equal to $\left[\begin{array}{ll}\vec{v}_{C} & \vec{i}\end{array}\right]^{\mathrm{T}}$, and $\mathbf{u}$ is input, equal to $\vec{i}_{w}, \vec{i}_{w}$ is determined by dc current and applied space vector of the CSC.

Based on continuous-time output model of CSC, discrete-time prediction model can be derived. Here the second-order Euler method is used to handle the second-order system in (9), which can be given as

$$
\left\{\begin{array}{l}
\mathbf{x}^{\mathbf{c}}(k+1)=\mathbf{x}(k)+T_{s}(\mathbf{A x}(k)+\mathbf{B u}(k)) \\
\mathbf{x}^{\mathbf{p}}(k+1)=\mathbf{x}^{\mathbf{c}}(k+1)+\frac{T_{s}}{2} \mathbf{A}\left(\mathbf{x}^{\mathbf{c}}(k+1)-\mathbf{x}(k)\right)
\end{array}\right.
$$

where $T_{s}$ is the sampling period in MPC, $\mathbf{x}(k)$ and $\mathbf{u}(k)$ are the state variable and input at $k$ th sampling instant. $\mathbf{x}^{\mathbf{c}}(k+1)$ is the predictor-corrector of state variable, and $\mathbf{x}^{\mathbf{p}}(k+1)$ is predicted state variable at $(k+1)$ th instant. With the information of state variable at $k$ th instant and selected space vector being applied during $k$ th sampling interval, state variable at $(k+1)$ th instant can be predicted based on (11). After some arrangements, (11) can be given as

$\mathbf{x}^{\mathbf{p}}(\mathbf{k}+\mathbf{1})=\boldsymbol{\Phi} \mathbf{x}(\mathbf{k})+\Gamma \mathbf{u}(\mathbf{k})$

Hence, with the space vector based SHE, discussed in Section III, load current vector at $(k+1)$ th instant can be predicted with output current vector following SHE-PWM pattern. The case in Fig. 9 (b) is used as an example. As Fig. 9 (b) shows, $\vec{I}_{6}$ and $\vec{I}_{1}$ are applied during the sampling interval. Here it is assumed that the dwell time for $\vec{I}_{6}$ is equal to $T_{1}$, then the effect of output current vector during the sampling interval can be given as

$T_{s} \cdot \mathbf{u}=T_{1} \cdot \vec{I}_{6}+\left(T_{s}-T_{1}\right) \cdot \vec{I}_{1}$

Based on (12) and (13), the load current vector at the end of the sampling interval can be predicted. A cost function is established here to evaluate the performance on reference track with SHE-PWM pattern, as

$J(k)=\frac{\left|\vec{i}^{\mathrm{ref}}-\vec{i}^{\mathrm{p}}(k+1)\right|}{\left|\vec{i}^{\mathrm{ref}}\right|}$

In (14), $\left|\vec{i}^{\mathrm{ref}}-\vec{i}^{\mathrm{p}}(k+1)\right|$ expresses the Euclidean distance between load current reference vector and predicted load

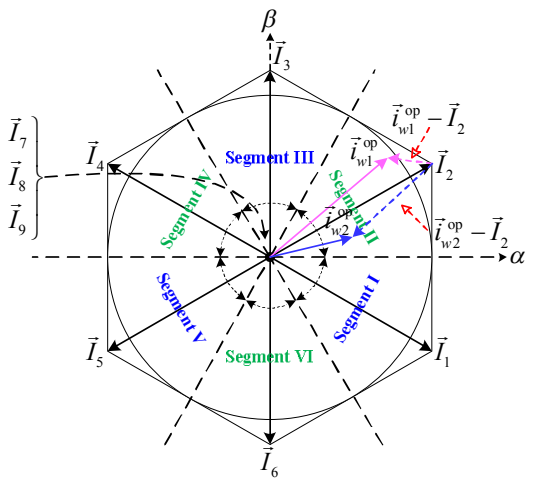

Fig. 11. Segment distribution on current vector domain.

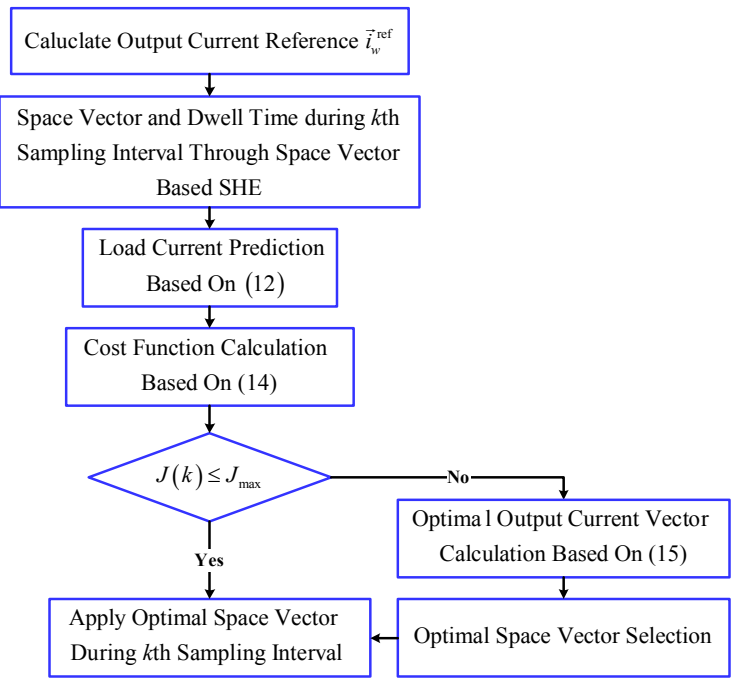

Fig. 12. Flowchart diagram of proposed MPSPC.

current vector. $J(k)$ represents a ratio between the Euclidean distance and the length of load current reference, which can be directly used to evaluate the performance on reference track.

Since SHE-PWM pattern cannot guarantee satisfactory performance during transients, a maximum limitation of the cost function, $J_{\max }$, can be defined, which is used as a criteria of transition between SHE-PWM pattern and MPC. When the value of $J(k)$ is smaller than $J_{\max }$, the performance on reference track with SHE-PWM pattern is acceptable, and output current of the CSC will follow SHE PWM pattern, which means that $\vec{i}_{w}$ is equal to $\vec{i}_{w}^{\text {SHE }}$. If $J(k)$ is larger than $J_{\max }$, the output current of the CSC during this sampling interval will be regulated through MPC approach. Here a dead-beat concept is introduced into the proposed MPSPC. Based on (12) and load current reference vector, optimal output current vector for the CSC, which eliminates the error between load current and its reference in just one sampling period, can be expressed as

$\vec{i}_{w}^{\text {op }}=\frac{\vec{i}^{\text {ref }}-\phi_{21} \cdot \vec{v}_{C}-\phi_{22} \cdot \vec{i}}{\varphi_{21} \cdot T_{s}}$

where $\phi_{21}, \phi_{22}$, and $\varphi_{21}$ denote elements in prediction matrices $\boldsymbol{\Phi}$ and $\boldsymbol{\Gamma}$. Here, unlike in traditional dead-beat control schemes, $\vec{i}_{w}^{\text {op }}$ is not synthesized based on SVM, since a 
high sampling frequency in MPSPC would lead to too large number of commutations during transients, which is not suitable to high power applications. To reduce the possible commutations, the space vector, which is the closest to $\vec{i}_{w}^{\text {op }}$, would be selected and applied during the whole sampling interval. In [20], this method is called low-complexity MPC, which preserves the advantage of conventional MPC, and simplifies the realization. According to the six active-state space vectors, the space vector domain can be divided into six segments, as Fig. 11 shows.

\section{Delay Compensation}

In ideal cases, the calculated optimal output current vector will be applied during the whole $k$ th sampling interval. However, in real implementation, calculation burden introduces a considerable time delay in the actuation, which will deteriorate the performance of MPSPC. A well-known two-step prediction method is used for delay compensation. The state variables at $(k+1)$ th instant need to be estimated based on the optimal output current vector selected for $k$ th sampling interval. After that, the load current vector at $(k+2)$ th instant will be predicted to optimally select the optimal output current vector for $(k+1)$ th sampling interval. At the time, the load current reference vector should be shifted one step forward. Finally, the cost function can be modified as

$J(k+1)=\frac{\left|\hat{\vec{i}}^{\text {ref }}-\vec{i}^{\mathrm{p}}(k+2)\right|}{\left|\hat{\vec{i}}^{\mathrm{ref}}\right|}$

where $\hat{\vec{i}}^{\text {ref }}$ is the load current reference vector for the $(k+2)$ th instant, and can be given as

$\hat{\vec{i}}^{\text {ref }}=\vec{i}^{\text {ref }} \cdot e^{j \omega T_{s}}$

in which $\omega$ is the angular rotating speed of the synchronous frame.

\section{SIMULATION RESULTS}

The simulation results for a $1 \mathrm{MW} / 4160 \mathrm{~V}$ CSC are presented in this section. The parameters used in simulation are listed in Table III. The proposed MPSPC with both six-pulse and eight-pulse SHE-PWM pattern are verified in simulation. The dc current is generated by an ideal dc current source, of which the value is kept at the rated dc current of 196 A. The sampling frequency of $10 \mathrm{kHz}$, meaning sampling interval of $100 \mu \mathrm{s}$ is used for the proposed MPSHEC. The value of $J_{\max }$ is selected as $15 \%$.

\section{A. Steady State Performance}

Table IV lists the steady state performance of proposed MPSPC at the operating condition of $i_{d}^{\text {ref }}$ equal to $196 \mathrm{~A}$ and $i_{q}^{\text {ref }}$ equal to 0 A. For comparison, the results of traditional
TABLE III

PARAMETERS IN SIMULATION AND EXPERIMENTS

\begin{tabular}{ccc}
\hline \hline Item & Simulation & Experiment \\
\hline Power rating & $1 \mathrm{MW}$ & $3 \mathrm{~kW}$ \\
Voltage rating & $4160 \mathrm{~V}$ & $208 \mathrm{~V}$ \\
Capacitor & $0.5 \mathrm{pu}$ & $0.6304 \mathrm{pu}$ \\
Filter inductor & $0.3 \mathrm{pu}$ & $0.2614 \mathrm{pu}$ \\
Load resistor & $0.3 \mathrm{pu}$ & $0.3467 \mathrm{pu}$ \\
\hline
\end{tabular}

TABLE IV

SIMULATED STEADY STATE PERFORMANCE

\begin{tabular}{cccccc}
\hline \multirow{2}{*}{ Control scheme } & SHE & \multicolumn{4}{c}{ Harmonic order } \\
\cline { 2 - 6 } & PATTERN & $5^{\text {th }}$ & $7^{\text {th }}$ & $11^{\text {th }}$ & $13^{\text {th }}$ \\
\hline \multirow{2}{*}{ Traditional SHE-MPC } & Six-pulse & $2.50 \%$ & $4.06 \%$ & $58.4 \%$ & $21.3 \%$ \\
& Eight-pulse & $1.66 \%$ & $1.98 \%$ & $3.06 \%$ & $36.3 \%$ \\
MPSPC & Six-pulse & $0.06 \%$ & $0.27 \%$ & $57.0 \%$ & $22.5 \%$ \\
& Eight-pulse & $0.28 \%$ & $0.06 \%$ & $0.16 \%$ & $36.8 \%$ \\
\hline \hline
\end{tabular}

SHE-MPC with quantization error are also provided, in which the sampling frequency is still $10 \mathrm{kHz}$. It can be found that with traditional SHE-MPC the unwanted low-order harmonics, no matter $5^{\text {th }}$ and $7^{\text {th }}$ for six-pulse waveform or $5^{\text {th }}, 7^{\text {th }}$, and $11^{\text {th }}$ for eight-pulse waveform, cannot be completely eliminated due to the effect of quantization errors. Whereas, MPSHEC totally completes the objectives of low-order harmonic elimination. Though a constant sampling frequency is used in MPSHEC, there is no compromise, caused by quantization error, between pre-calculated optimal switching angles and finally applied ones.

\section{B. Transient Responses}

Compared to only space vector based SHE without prediction process, the proposed MPSPC improves dynamic performance. For comparison, transient responses with only space vector based SHE are provided, either. In Fig. 13, the output frequency of the CSC is $60 \mathrm{~Hz}$, and six-pulse SHE waveform is used. At time $t$ of $0.1 \mathrm{~s}, i_{s d}^{\text {ref }}$ is dropped from $196 \mathrm{~A}$ to only $39.3 \mathrm{~A} . i_{s q}^{\text {ref }}$ is kept at 0 during the whole process. During the transient process, the settling time, $T_{\text {settle }}$, of load current is measured with the response stay in a range of $5 \%$ of the rated value. It can be observed from Fig. 13 (a) that the transient responses of load currents are very sluggish, and $T_{\text {settle }}$ is equal to $23 \mathrm{~ms}$ when with only space vector based SHE. Since capacitor and filter inductor construct a LC resonant tank, significant resonance can be observed in load currents, especially in $d q$-axis components. With MPSPC, during transients, since the calculated cost function can be easily higher than $J_{\text {max }}$, the PWM pattern of the CSC is naturally changed from SHE waveform to optimally selected space vectors by MPC. With this mechanism, the load currents fast track their references, and $T_{\text {settle }}$ is reduced to only $14 \mathrm{~ms}$. Moreover, the LC resonance is somewhat suppressed, since the calculated optimal output current reference forces load currents to follow their references. After transients, it can be found that output PWM pattern returns to SHE waveform due to the reduced cost function value in steady state. Hence, without the 

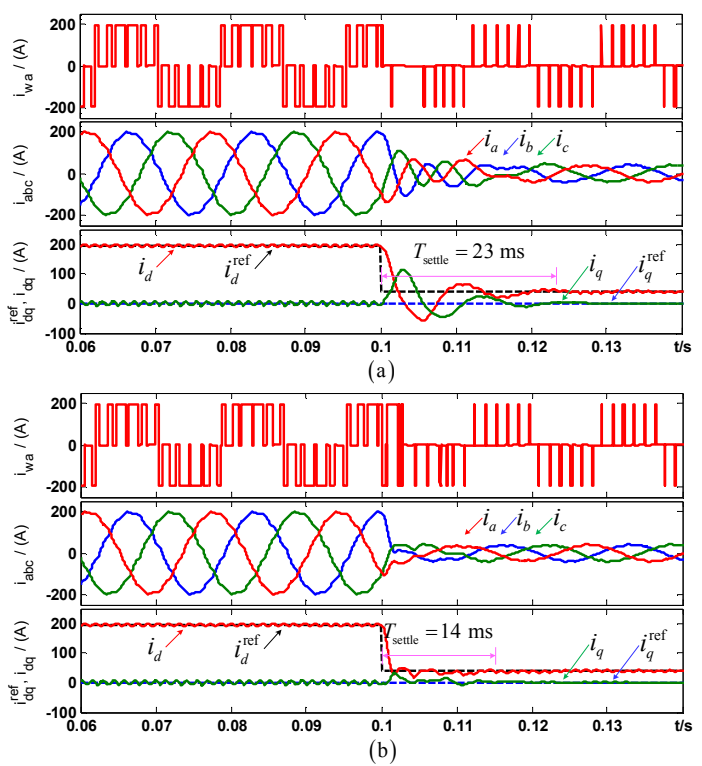

Fig. 13. Transient responses with six-pulse SHE waveform: (a) space vector based SHE and (b) MPSPC.
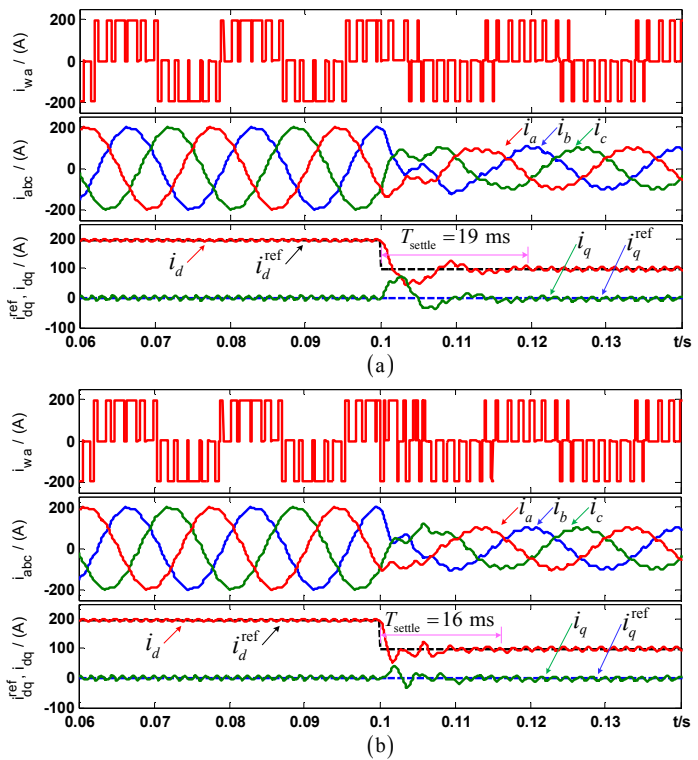

Fig. 14. Transient performance during output frequency change: (a) space vector based SHE and (b) MPSPC.

weighting factor tuning issue in the cost function, the proposed MPSPC can still keep SHE waveform in steady state, and improves the dynamic performance during transients.

\section{EXPERIMENTS RESULTS}

The experimental verification of proposed MPSPC with six-pulse and eight-pulse SHE-PWM patterns is also conducted. The parameters used in experiments have already been listed in Table III. The sampling frequency is still $10 \mathrm{kHz}$, meaning the sampling interval of $100 \mu \mathrm{s}$, and $J_{\max }$ is still $15 \%$ as in simulation. The dc current is kept at $11.8 \mathrm{~A}$, which is the rated value for a $3 \mathrm{~kW} / 208 \mathrm{~V} \mathrm{CSC}$.

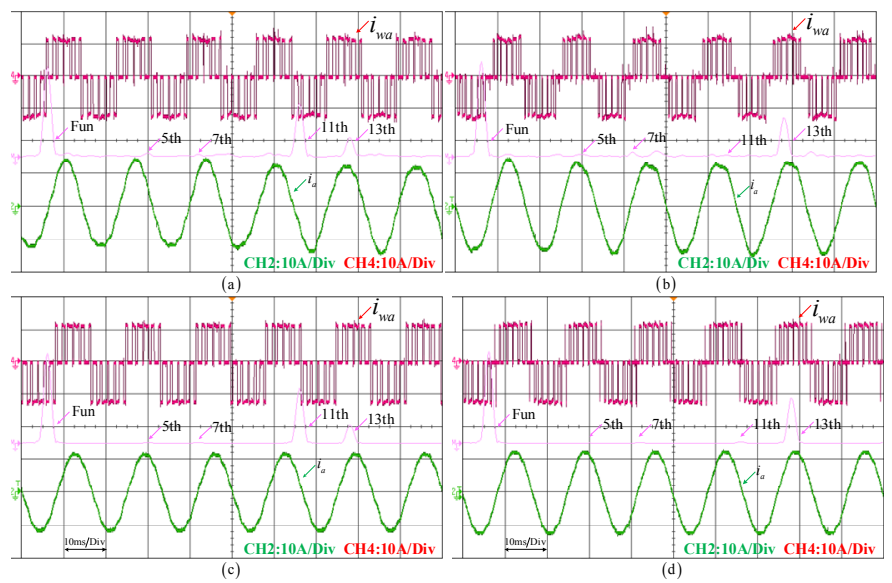

Fig. 15. Spectrum diagram of experimental output PWM currents: (a) SHE-MPC with six-pulse waveform, (b) SHE-MPC with eight-pulse waveform, (c) MPSPC with six-pulse waveform, and (d) MPSPC with eight-pulse waveform.

TABLE V EXPERIMENTAL STEADY STATE PERFORMANCE

\begin{tabular}{cccccc}
\hline \hline \multirow{2}{*}{ Control scheme } & SHE & \multicolumn{4}{c}{ Harmonic order } \\
\cline { 3 - 6 } & PATTERN & $5^{\text {th }}$ & $7^{\text {th }}$ & $11^{\text {th }}$ & $13^{\text {th }}$ \\
\hline Traditional & Six-pulse & $6.36 \%$ & $2.73 \%$ & $60.0 \%$ & $20.0 \%$ \\
SHE-MPC & Eight-pulse & $2.63 \%$ & $5.26 \%$ & $2.61 \%$ & $42.1 \%$ \\
\multirow{2}{*}{ MPSPC } & Six-pulse & $0.91 \%$ & $0.91 \%$ & $61.8 \%$ & $20.0 \%$ \\
& Eight-pulse & $0.45 \%$ & $0.91 \%$ & $1.81 \%$ & $50.9 \%$ \\
\hline \hline
\end{tabular}

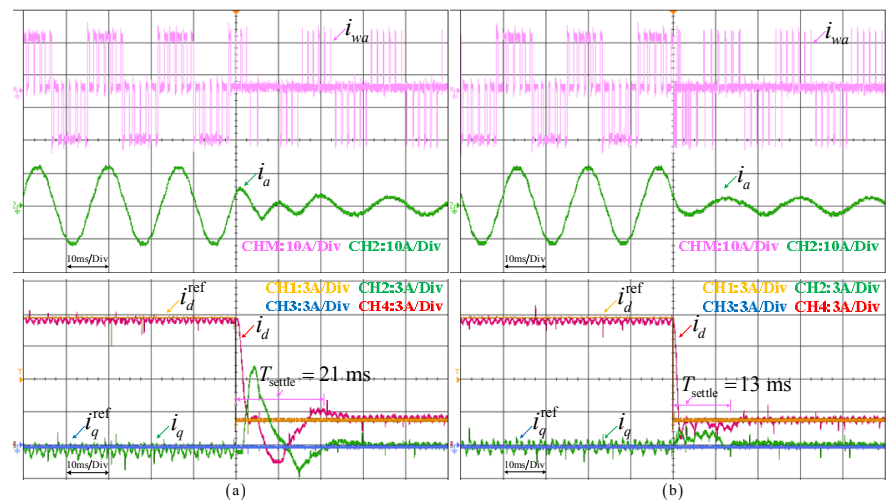

Fig. 16. Experimental transient responses with six-pulse SHE waveform: (a) space vector based SHE and (b) MPSPC.

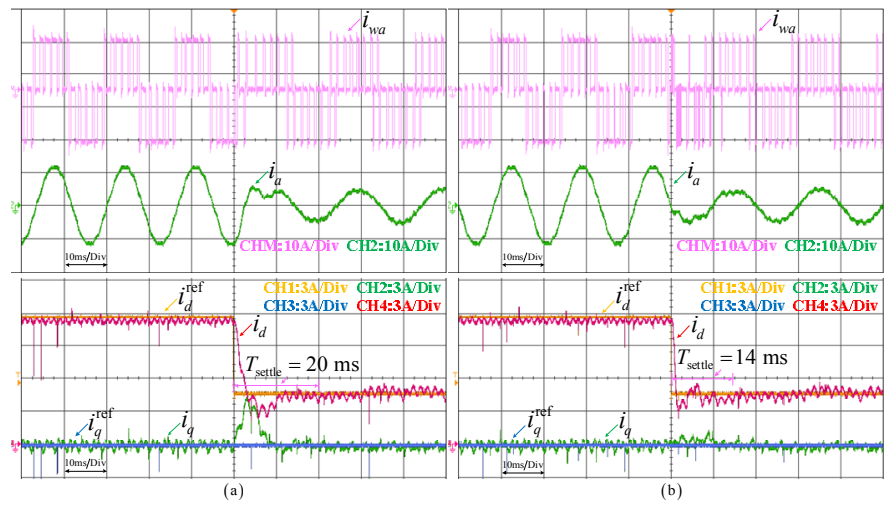

Fig. 17. Experimental transient performance during output frequency change: (a) space vector based SHE and (b) MPSPC.

A. Steady State Performance

The experimental results of both traditional SHE-MPC and 
MPSPC are presented at operating condition of $i_{d}^{\text {ref }}$ equal to $11.8 \mathrm{~A}$ and $i_{q}^{\text {ref }}$ equal to $0 \mathrm{~A}$. Fig. 15 shows phase A load current, output PWM current, and its spectrum diagram of traditional SHE-MPC and MPSPC. Due to quantization error, the output PWM current of traditional SHE-MPC contains obvious low-order harmonics. Moreover, as can be observed in Fig. 15 (b), the quantization effect makes some very small pulses missed from eight-pulse waveform, which leads to only six or seven pulses per half cycle. On the other hand, MPSPC achieves better performance on low-order harmonic elimination than traditional SHE-MPC, no matter with six-pulse or eight-pulse SHE-PWM pattern. The details on the low-order harmonic contents are listed in Table V. With proposed MPSPC, the unwanted low-order harmonics can be mitigated to very small values. It can be found that the harmonic contents in Table $\mathrm{V}$ are higher than the results presented in Table VI. The reason is that the dc current used in experiments is not ideal, and contains some ripples, which causes some compromises on low-order harmonic elimination.

\section{B. Transient Response}

Fig. 16 shows the experimental transient responses with six-pulse SHE-PWM waveform. The output frequency is 60 $\mathrm{Hz}$, and $i_{d}^{\text {ref }}$ suddenly drops from $11.8 \mathrm{~A}$ to $2.36 \mathrm{~A}$. From top to bottom, phase A output PWM current, load current, $d q$-axe load current components, and their respective references are shown. With only space vector based SHE, significant resonance can be observed in the waveforms of $i_{d}$ and $i_{q}$, as Fig. 16 (a) shows. The settling time is equal to $21 \mathrm{~ms}$. In Fig. 16 (b), with MPSPC, $i_{d}$ changes from $11.8 \mathrm{~A}$ to $2.36 \mathrm{~A}$ faster, and the resonance in load current is suppressed, with the decrease of the settling time to $13 \mathrm{~ms}$. The transition from SHE-PWM to MPC can be found from the output PWM current during the transient. After the transient, the output PWM current is governed by SHE-PWM again.

\section{CONCLUSION}

In this work, MPSPC scheme based on a proposed space-vector-based SHE method is presented, which achieves low-order harmonics elimination in steady state, and improves dynamic performance. In MPSPC, the predictive controller modifies the optimal output current vector during transients. Regarding the steady state, MPSPC is able to resemble the optimized PWM current pattern, which follows the SHE-PWM pattern. As evidenced by the results, compared to the state-of-the-art schemes, the proposed scheme offers three major advantages:

1) No Quantization Error: A space vector based SHE generation method is proposed as the foundation of MPSPC, which eliminates the quantization effect introduced by a constant sampling frequency for MPC. Without quantization error, the low-order harmonics elimination based on pre-calculated optimal switching angles can be thoroughly completed.
2) No Weighting Factor: Another benefit offered by MPSPC is that there is no weighting factor used in it. Though a maximum limitation of tracking error needs to be selected to ensure the natural transition between SHE-PWM pattern and optimal output current vector selected by MPC during transients, it owns more significant physical meaning in comparison with a weighting factor.

3) On-Step Prediction: MPSPC only uses a one-step or horizon-one prediction, which leads to a simpler structure, and reduces the complexity, compared to existing multi-step schemes.

\section{REFERENCES}

[1] B. Wu, High-power converters and AC drives: John Wiley \& Sons, 2006.

[2] B. Wu, J. Pontt, J. Rodriguez, S. Bernet, and S. Kouro, "Current-Source Converter and Cycloconverter Topologies for Industrial Medium-Voltage Drives," Industrial Electronics, IEEE Transactions on, vol. 55, pp. 2786-2797, 2008.

[3] Z. Wang, B. Wu, D. Xu, and N. R. Zargari, "Hybrid PWM for High-Power Current-Source-Inverter-Fed Drives With Low Switching Frequency," Power Electronics, IEEE Transactions on, vol. 26, pp. 1754-1764, 2011.

[4] J. C. Wiseman and B. Wu, "Active damping control of a high-power PWM current-source rectifier for line-current THD reduction," Industrial Electronics, IEEE Transactions on, vol. 52, pp. 758-764, 2005.

[5] Y. W. Li, B. Wu, N. R. Zargari, J. C. Wiseman, and D. Xu, "Damping of PWM Current-Source Rectifier Using a Hybrid Combination Approach," Power Electronics, IEEE Transactions on, vol. 22, pp. 1383-1393, 2007.

[6] S. Kouro, P. Cortes, R. Vargas, U. Ammann, and J. Rodriguez, "Model Predictive Control - A Simple and Powerful Method to Control Power Converters," Industrial Electronics, IEEE Transactions on, vol. 56, pp. 1826-1838, 2009.

[7] S. Kouro, M. A. Perez, J. Rodriguez, A. M. Llor, and H. A. Young, "Model Predictive Control: MPC's Role in the Evolution of Power Electronics," IEEE Industrial Electronics Magazine, vol. 9, pp. 8-21, 2015.

[8] V. Yaramasu, B. Wu, and J. Chen, "Model-Predictive Control of Grid-Tied Four-Level Diode-Clamped Inverters for High-Power Wind Energy Conversion Systems," Power Electronics, IEEE Transactions on, vol. 29, pp. 2861-2873, 2014.

[9] Y. C. Zhang and H. T. Yang, "Model Predictive Torque Control of Induction Motor Drives With Optimal Duty Cycle Control," Power Electronics, IEEE Transactions on, vol. 29, pp. 6593-6603, 2014.

[10] Y. C. Zhang and H. T. Yang, "Generalized Two-Vector-Based Model-Predictive Torque Control of Induction Motor Drives," Power Electronics, IEEE Transactions on, vol. 30, pp. 3818-3829, 2015.

[11] M. Rivera, S. Kouro, J. Rodriguez, B. Wu, and J. Espinoza, "Predictive control of a current source converter operating with low switching frequency," in IECON 2012 - 38th Annual Conference on IEEE Industrial Electronics Society, 2012, pp. 674-679.

[12] P. Zavala, M. Rivera, S. Kouro, J. Rodriguez, B. Wu, V. Yaramasu, et al., "Predictive control of a current source rectifier with imposed sinusoidal input currents," in Industrial Electronics Society, IECON 2013 - 39th Annual Conference of the IEEE, 2013, pp. 5842-5847.

[13] P. Correa, J. Rodriguez, I. Lizama, and D. Andler, "A Predictive Control Scheme for Current-Source Rectifiers," Industrial Electronics, IEEE Transactions on, vol. 56, pp. 1813-1815, 2009.

[14] T. Geyer, G. Papafotiou, and M. Morari, "Model Predictive Direct Torque Control-Part I: Concept, Algorithm, and Analysis," Industrial Electronics, IEEE Transactions on, vol. 56, pp. 1894-1905, 2009.

[15] T. Geyer, N. Oikonomou, G. Papafotiou, and F. Kieferndorf, "Model predictive pulse pattern control," in Energy Conversion Congress and Exposition (ECCE), 2011 IEEE, 2011, pp. 3306-3313.

[16] T. Geyer and D. E. Quevedo, "Performance of Multistep Finite Control Set Model Predictive Control for Power Electronics," Power Electronics, IEEE Transactions on, vol. 30, pp. 1633-1644, 2015.

[17] S. Kouro, B. L. Rocca, P. Cortes, S. Alepuz, B. Wu, and J. Rodriguez, "Predictive control based selective harmonic elimination with low switching frequency for multilevel converters," in 2009 IEEE Energy Conversion Congress and Exposition, 2009, pp. 3130-3136. 
[18] R. Aguilera, P. Acuna, P. Lezana, G. Konstantinou, B. Wu, S. Bernet, et al., "Selective Harmonic Elimination Model Predictive Control for Multilevel Power Converters," IEEE Transactions on Power Electronics, vol. PP, pp. 1-1, 2016.

[19] J. Napoles, J. I. Leon, R. Portillo, L. G. Franquelo, and M. A. Aguirre, "Selective Harmonic Mitigation Technique for High-Power Converters," IEEE Transactions on Industrial Electronics, vol. 57, pp. 2315-2323, 2010.

[20] Y. C. Zhang and W. Xie, "Low Complexity Model Predictive Control Single Vector-Based Approach," Power Electronics, IEEE Transactions on, vol. 29, pp. 5532-5541, 2014.

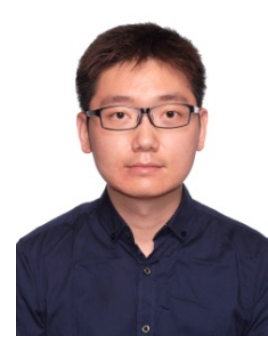

Hang Gao received the B.Sc and M.A.Sc degrees in electrical engineering from Hefei University of Technology (HFUT), Anhui, China, respectively in 2011 and 2014. He is currently working towards the $\mathrm{Ph} . \mathrm{D}$. degree in electrical and computer engineering at Ryerson University, Toronto, ON, Canada. His research interests include high-power converter topologies, model predictive control, and ac motor drives. Mr. Gao is a recipient of Ontario Trillium Scholarship (OTS), for pursuing his Ph. D. study in power electronics.

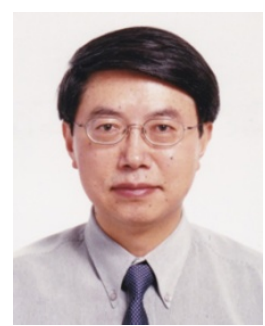

Bin Wu (S'89-M'92-SM'99-F'08) received his M.A.Sc. and Ph.D. degrees in electrical and computer engineering from the University of Toronto, Canada in 1989 and 1993, respectively. He joined Ryerson University in 1993, where he is currently a Professor and Senior NSERC/Rockwell Automation Industrial Research Chair in Power Electronics and Electric Drives. Dr. Wu has published more than 350 technical papers, authored/coauthored two Wiley-IEEE Press books, and holds more than 30 granted/pending US/European patents in the area of power conversion, medium voltage drives, and renewable energy systems. Dr. Wu received the Gold Medal of the Governor General of Canada in 1993, Premier's Research Excellence Award in 2001, NSERC Synergy Award for Innovation in 2002, Ryerson Distinguished Scholar Award and Professional Engineers Ontario (PEO) Engineering Excellence Medal in 2014. He is a fellow of Engineering Institute of Canada (EIC) and Canadian Academy of Engineering (CAE).

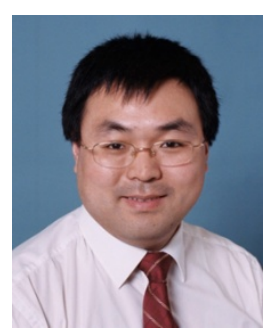

Dewei(David) Xu (S'99-M'01) received the B.Sc, M.A.Sc, and Ph.D. degree in electrical engineering from Tsinghua University, Bejing, China, respectively in 1996, 1998, and 2001. He has been working in Ryerson University, Toronto, Ontario since 2001, where he is currently Professor. His research interests include renewable energy system, high power converters, electric motor drives and advanced digital control for power electronics.

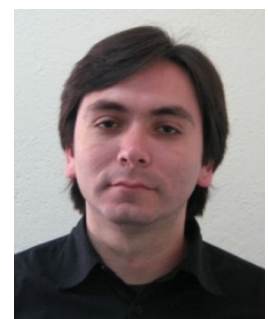

Ricardo P. Aguilera (S'01-M'12) received his M.Sc. degree in Electronics Engineering from the Universidad Tecnica Federico Santa Maria (UTFSM), Chile, 2007, and Ph.D. degree in Electrical Engineering from The University of Newcastle (UoN), Australia, 2012. From 2012 to 2013, he was a Research Academic at UoN, where he was part of the Centre for Complex Dynamic Systems and Control. From 2014 to 2016, he was a Senior Research Associate at The University of New South Wales (UNSW), Australia, where he was part of the Australian Energy Research Institute (AERI). Since 2016, he is with the School of Electrical, Mechanical and Mechatronic Systems, at The University of Technology Sydney (UTS), Australia, where he currently holds a Lecturer position. His main research interests include power electronics, and theoretical and practical aspects on model predictive control.
Pablo Acuña (M'12) received the B.Sc. in Electronics Engineering, the Electronics Engineering Professional degree, and the Ph.D. degrees in Electrical Engineering from the University of Concepcion, Chile, in 2004, 2007, and 2013 respectively. He is currently Research Associate at the school of Electrical Engineering and Telecommunications, University of New South Wales, Australia. His research interests include electrical power conversion systems and its applications to industry, transportation and utility. 